\title{
Multi-functional bismuth-doped bioglasses: combining bioactivity and photothermal response for bone tumor treatment and tissue repair
}

\author{
Liping Wang ${ }^{1}$, Nicholas J. Long ${ }^{2}$, Lihua Li ${ }^{1}$, Yao Lu ${ }^{3}$, Mei Li ${ }^{3}$, Jiangkun Cao ${ }^{1}$, Yu Zhang ${ }^{3}$, Qinyuan Zhang ${ }^{1}$, Shanhui Xu' \\ Zhongmin Yang ${ }^{1}$, Chuanbin Mao ${ }^{4,5}$ and Mingying Peng ${ }^{1}$
}

\begin{abstract}
Treatment of large bone defects derived from bone tumor surgery is typically performed in multiple separate operations, such as hyperthermia to extinguish residual malignant cells or implanting bioactive materials to initiate apatite remineralization for tissue repair; it is very challenging to combine these functions into a material. Herein, we report the first photothermal (PT) effect in bismuth (Bi)-doped glasses. On the basis of this discovery, we have developed a new type of Bi-doped bioactive glass that integrates both functions, thus reducing the number of treatment cycles. We demonstrate that Bi-doped bioglasses (BGs) provide high PT efficiency, potentially facilitating photoinduced hyperthermia and bioactivity to allow bone tissue remineralization. The PT effect of Bi-doped BGs can be effectively controlled by managing radiative and non-radiative processes of the active Bi species by quenching photoluminescence (PL) or depolymerizing glass networks. In vitro studies demonstrate that such glasses are biocompatible to tumor and normal cells and that they can promote osteogenic cell proliferation, differentiation, and mineralization. Upon illumination with near-infrared (NIR) light, the bioglass (BG) can efficiently kill bone tumor cells, as demonstrated via in vitro and in vivo experiments. This indicates excellent potential for the integration of multiple functions within the new materials, which will aid in the development and application of novel biomaterials.
\end{abstract}

\section{Introduction}

Bone is a complex type of tissue with the capacity to self-repair ${ }^{1}$. However, regeneration may fail for large bone defects, such as those caused by bone cancer or pathological fractures. For example, common treatments for bone cancer currently involve surgery, chemotherapy, and radiotherapy ${ }^{2}$. These treatments may harm healthy cells

\footnotetext{
Correspondence: Mingying Peng (pengmingying@scut.edu.cn)

${ }^{1}$ The State Key Laboratory of Luminescent Materials and Devices, Guangdong Engineering Technology Research and Development Center of Special Optical Fiber Materials and Devices, Guangdong Provincial Key Laboratory of Fiber Laser Materials and Applied Techniques, School of Materials Science and Engineering, South China University of Technology, 510641 Guangzhou, China ${ }^{2}$ Department of Chemistry, Imperial College London, South Kensington, London SW7 2AZ, UK

Full list of author information is available at the end of the article
}

in the vicinity of the defect, causing inevitable and permanent defects in the bone tissue. An additional problem can be the presence of residual malignant cells, which may lead to tumor recurrence, significantly affecting treatment prognosis. Therefore, treatments often involve complex secondary surgical operations in which large amounts of bone tissue are removed ${ }^{3}$. This plethora of problems, therefore, calls for multi-functional bioactive materials to aid in tumor treatment and tissue regeneration.

Compared to traditional treatment methods, hyperthermia has been proposed as a minimally invasive way to shorten patient recovery times ${ }^{4}$. It involves the use of functional nanoparticles, laser-induced thermal therapy, or high-intensity ultrasonic treatment ${ }^{5-8}$. In an interesting combination of functions, magnetic bioceramics have

\section{(c) The Author(s) 2018}

\footnotetext{
(c) Open Access This article is licensed under a Creative Commons Attribution 4.0 International License, which permits use, sharing, adaptation, distribution and reproduction cc in any medium or format, as long as you give appropriate credit to the original author(s) and the source, provide a link to the Creative Commons license, and indicate if changes were made. The images or other third party material in this article are included in the article's Creative Commons license, unless indicated otherwise in a credit line to the material. If material is not included in the article's Creative Commons license and your intended use is not permitted by statutory regulation or exceeds the permitted use, you will need to obtain permission directly from the copyright holder. To view a copy of this license, visit http://creativecommons.org/licenses/by/4.0/.
} 
been proposed. They combine thermal activity for application in hyperthermia and bioactivity for apatite mineralization, leading to bone tissue repair ${ }^{9}$. For the latter, bioactive glasses have become an attractive alternative, as they provide significantly higher compositional variability and offer the potential for tailored reactivity ${ }^{10}$. Bioceramics and bioglasses (BGs) exhibiting both magnetism and hydroxyapatite (HAp) formation have been investigated for potential application on bone tumors through magnetic hyperthermia ${ }^{11}$. However, various fundamental aspects, such as exposure time, material compatibility, and toxicity of $\mathrm{Fe}_{2} \mathrm{O}_{3}$ - or $\mathrm{FeO}$-based approaches, need to be addressed before clinical application can be considered $^{12}$. In addition to magnetic hyperthermia, photothermal (PT) therapy induces thermal apoptosis through heat generated from optical input. Here, the key component is the employed PT conversion agent, which absorbs and converts near-infrared (NIR) light into heat. Various nanoparticle species (NPs) are currently considered as PT agents $^{13-16}$, including, most prominently, metallic NPs but also carbon-based NPs, semiconductor NPs, and organic compounds. In metallic NPs, the thermal effect is generated by localized surface plasmon resonance, which depends on metal or alloy species, particle size, shape, and number density ${ }^{17-19}$. For example, varying the particle shape of $\mathrm{Au}$ between rods and shell structures enables a shift in the active spectral range from visible light to NIR; however, this is at the expense of conversion efficiency $^{20,21}$. Carbon-based NPs include carbon nanotubes, carbon nanodots and graphene, which, in addition to having PT functionality, are biocompatible ${ }^{22-24}$. In carbon nanotubes, similar to metallic NPs, light-to-heat conversion is due to the light-induced collective motion of free carriers, that is, excitation of the $\pi$-plasmon. Problematic to application in hyperthermia, the oxidization process, which is meant to enable dispersion in water, impairs PT efficiency $^{25,26}$.

In the third group of PT materials, semiconductor NPs often provide a significantly higher conversion efficiency than others such as $\mathrm{Au}$ NPs. For example, $\mathrm{Cu}_{2-x} \mathrm{Se}$ or $\mathrm{Cu}_{9} \mathrm{~S}_{5}$ nanocrystals produce PT heating with a conversion efficiency of $22 \%^{27}$ and $25.7 \%^{28}$, respectively. The main limitation is their well-documented toxicity, especially considering the expectedly high retention time in the body ${ }^{29}$. Furthermore, the spectral mechanism of PT remains unclear; it is either NIR absorption through interband electronic transitions or broadband carriers, and this uncertainty makes dedicated tailoring of properties difficult $^{30}$. Organic compounds have also been considered as an alternative with comparably high biocompatibility and biodegradability. Here, problems are primarily related to the poor PT stability ${ }^{31,32}$.

None of these previous strategies, however, can simultaneously integrate PT and bioactivity into one material.
Here, we have discovered the first PT effect in bismuth (Bi)-doped glasses. On the basis of this finding, we have developed a new model of Bi-doped bioglass (BG) that simultaneously combines both functions. Generally, once light is absorbed by an optical material, it is very challenging to control radiative and non-radiative processes, which eventually evolve into photoluminescence (PL) and heat, respectively. When heat is accumulated within the matrix, the effect of PT gradually emerges. We find that depolymerizing the matrix glass network can suppress the spontaneous radiation process following NIR excitation; thus it enhances PT conversion efficiency.

In vitro and in vivo experiments were performed to evaluate the biocompatibility, HAp formation, and PT therapeutic ability of Bi-doped BGs. These experiments were designed to investigate the biocompatibility of glasses with both tumor and normal cells and how they promote osteogenic cell proliferation, differentiation, and mineralization on the surface of samples. Under irradiation with NIR light, in vivo experiments with nude mice showed that the materials can efficiently kill bone tumor cells. The model type of Bi-doped BGs can be heated to a desirable temperature range from 38 to $86^{\circ} \mathrm{C}$ by either glass components or incident laser power density, and they are bioactive and resorptive. Therefore, they enable remineralization of apatite to facilitate bone regeneration.

\section{Materials and methods}

\section{Sample preparation}

All glass samples were prepared by melting and quenching, and the melting conditions were adapted to the respective glasses. Sample preparation and processing procedures are summarized in the supplementary information, with detailed nominal compositions and melting conditions listed in Table S1. Glass sample codes are shown in column 2 of Table S1. For example, G5AxB indicates $(95-x) \mathrm{GeO}_{2}-5 \mathrm{Al}_{2} \mathrm{O}_{3}-x \mathrm{Bi}_{2} \mathrm{O}_{3}(x=0.05,0.5,1.5$, $2,4,6,10)$. All the glass samples were free of bubbles. The sample without Bi appears colorless. Generally, the sample color becomes deeper as the Bi content increases. For example, the germanate glass sample is purple-red when $x=0.05$, and it becomes reddish brown and even deep reddish brown when $x=2$ and $x=10$, respectively. Similar to Bi-doped germanate samples, Bi-doped silicate glass appear reddish brown, while Bi-doped phosphosilicate is light brown. A portion of the glass samples were ground, milled, and sieved using 400 mesh for consequent biological experiments.

\section{Materials characterization}

The PT effect was evaluated by the setup shown in Figure S1, where an $808 \mathrm{~nm}$ laser diode (LD) and a $\mathrm{ZnSe}$ infrared thermometer (LumaSense IMPAC, Germany), respectively, were used to illuminate samples and monitor 


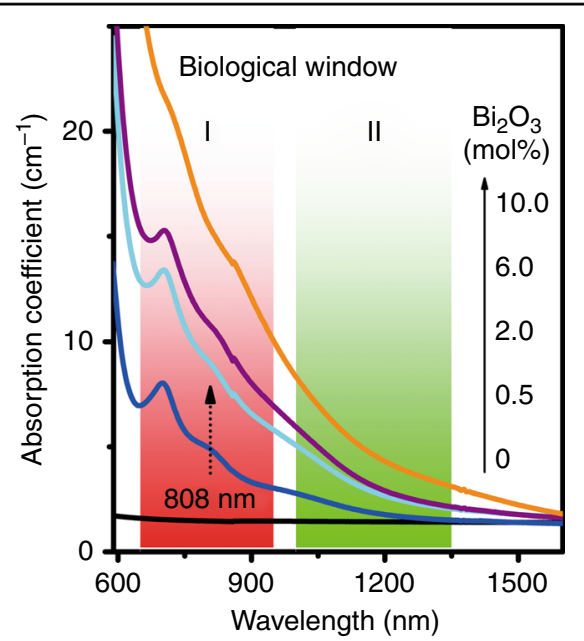

Fig. 1 UV to NIR absorption spectra of Bi-doped germanate glass G5AxB as a function of dopant content $x$ (labels, mol\%). For reference, the first and second biological windows are shaded in red and green, respectively.

the temperature of the sample surface in air in a range from 273 to $1173 \mathrm{~K}$. Here, the $808 \mathrm{~nm}$ laser was selected as the irradiation source to avoid water absorption in tissues and improve the tissue-penetration depth ${ }^{33,34}$. The temperature of the samples immersed in simulated body fluid (SBF) solution was measured with a digital thermometer (Kangyou, Nanjing, China). Optical absorption spectra were measured with a Perkin Elmer Lambda 900 UV/Vis/NIR spectrophotometer in a spectral range from 200 to $3200 \mathrm{~nm}$. PL spectra were recorded with a Zolix Omni $\lambda 3007$ spectrometer equipped with an InGaAs photodetector and a SR830 Stanford lock-in amplifier. The fluorescence lifetimes were measured with a digital phosphor oscilloscope (TDS3012C, Tektronix, America). The excitation spectra were measured with an Edinburgh Instrument FLS 920 (Livingston, WL, UK) equipped with a liquid nitrogen-cooled photomultiplier (Hamamatsu R5509-72, Hamamatsu, Japan).

\section{Biological experiments}

Two representative normal cells, namely, mouse fibroblast (L929) ${ }^{35}$ and murine pre-osteoblast (MC3T3-E1) ${ }^{36}$ cell lines, and two types of tumor cells, namely, rat osteosarcoma-derived (UMR106) ${ }^{37}$ and human osteosarcoma (U2OS) ${ }^{38}$ cells, were purchased from the Type Culture Collection of the Chinese Academy of Sciences. The details are summarized in the supplementary information on cell culture, including in vitro biocompatibility; in vitro mineralization of HAp on BG surfaces in SBF; adhesion, proliferation, differentiation, and mineralization of murine pre-osteoblast cells on Bi-doped BGs; in vitro PT performance of Bi-doped BGs; and in vivo PT therapy experiments on nude mice. All animal studies were approved by the Institutional Animal Care and Use Committee (IACUC) of Guangzhou General Hospital of Guangzhou Military Command. Adult male Balb/c nude mice were purchased from Medical Experimental Animal Center of Guangdong Province.

\section{Results and discussion}

Discovery of PT effect in Bi-doped germanate glasses

Typical materials for PT therapy applications should exhibit NIR absorption peaks within the first and second biological transparent windows (Fig. 1) to combine maximum radiation penetration into the tissue with optimal light absorption of the converter species ${ }^{17,39,40}$. Hence, the overall energy input can be minimized, reducing possible damage to normal tissues during therapy. We occasionally found that, when irradiated by $808 \mathrm{~nm} \mathrm{LD}$, all Bi-doped germanate glasses became very hot during investigations of the PL properties. These doped glasses had absorption peaks at $\sim 700 \mathrm{~nm}, \sim 800 \mathrm{~nm}$, and $\sim 1000 \mathrm{~nm}$ that were clearly absent in the undoped sample G5A0B (Fig. 1). These peaks, according to previous reports, can be assigned to the transition of $\mathrm{Bi}^{+}$from ${ }^{3} \mathrm{P}_{0}$ to ${ }^{3} \mathrm{P}_{2}$, and the transitions of $\mathrm{Bi}^{0}$ from ${ }^{4} \mathrm{~S}_{3 / 2}$ to ${ }^{2} \mathrm{D}_{3 / 2}(2)$ and ${ }^{2} \mathrm{D}_{3 / 2}(1)$, respectively ${ }^{41,42}$. The absorption coefficient at $808 \mathrm{~nm}$ increases gradually from $\sim 4.9 \mathrm{~cm}^{-1}$ to $\sim 15.4 \mathrm{~cm}^{-1}$ with an increase in the $\mathrm{Bi}_{2} \mathrm{O}_{3}$ concentration from 0.5 to $10 \mathrm{~mol}$ $\%$. Thus, $\mathrm{Bi}_{2} \mathrm{O}_{3}$ addition triggers the desired NIR absorptions, which are primarily located in the first and second biological windows (Fig. 1). This is a prerequisite for PT conversion.

To quantify the PT effect, we set up a device, as illustrated in Figure S1, to detect the surface temperature of Bi-doped germanate glass samples G5AxB (for sample codes, see Table S1). Samples were continuously irradiated until they reached a steady thermal state, followed by cooling to room temperature upon laser shut-off. When samples were irradiated by the $808 \mathrm{~nm}$ laser, the temperature of G5A2B increased to $54,93,126,155,183$, 205 , and $223^{\circ} \mathrm{C}$ as the incident power density increased to $0.3,1.1,1.8,2.5,3.3,4$, and $4.6 \mathrm{~W} \mathrm{~cm}^{-2}$, respectively. In contrast, under the same conditions of laser irradiation, significant temperature variation did not appear in the blank sample, G5A0B. For example, at a power density of $1.1 \mathrm{~W} \mathrm{~cm}^{-2}$, the temperature of sample G5A2B increases approximately linearly from $37^{\circ} \mathrm{C}$ (simulating human body temperature) to $73^{\circ} \mathrm{C}$ at a rate of $4.6{ }^{\circ} \mathrm{C} \mathrm{s}^{-1}$ in the first $10 \mathrm{~s}$ (red line, inset (a) of Fig. 2). Subsequently, the temperature reaches a plateau at $\sim 93{ }^{\circ} \mathrm{C}$. In comparison, $\mathrm{Au}$ nanoshells, or nanorods, have been reported to provide a typical temperature increase of $15^{\circ} \mathrm{C}$ and $22^{\circ} \mathrm{C}$, respectively, after $5 \mathrm{~min}$ irradiation under similar illumination conditions $\left(1 \mathrm{~W} \mathrm{~cm}^{-2}, 800 \mathrm{~nm}\right)^{27}$. In the present case, a steady state was reached after $\sim 90 \mathrm{~s}$; however, this depended on sample geometry and the heat exchange 


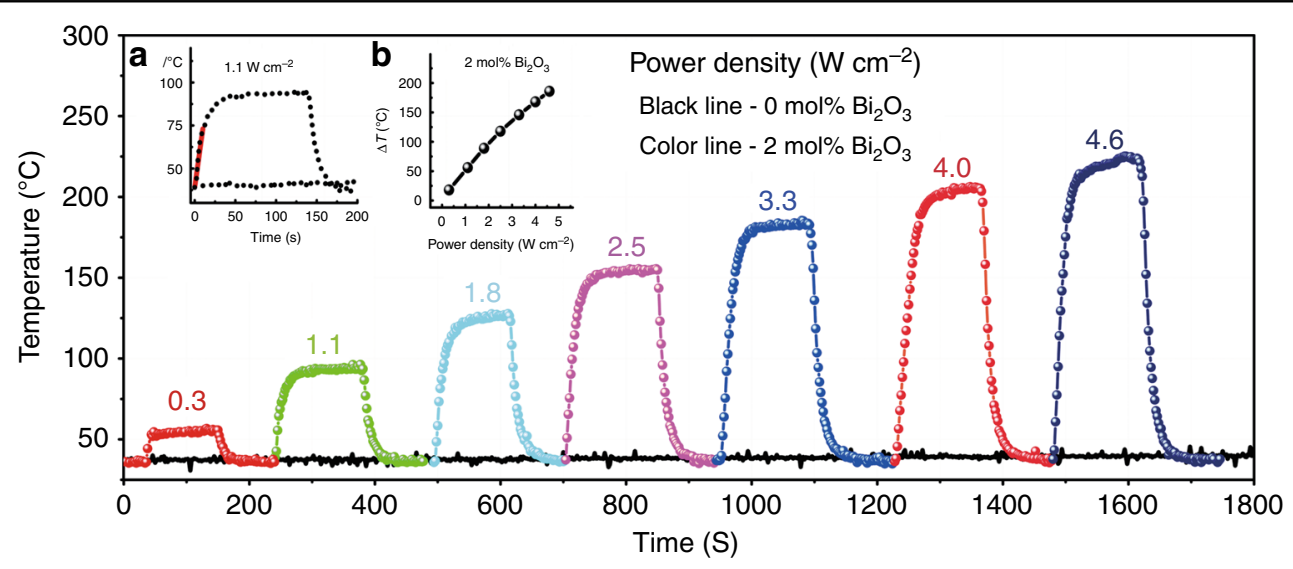

Fig. 2 Discovery of PT effect in Bi-doped germanate glasses. Temperature of glass sample G5A2B (colored lines) and blank reference sample G5AOB (black line) as a function of irradiation time and incident power density (label, in $\mathrm{W} \mathrm{cm}^{-2}$ ) of CW $808 \mathrm{~nm}$ LD. Each curve represents the average of seven on/off cycles. Inset $\mathbf{a}$ shows a magnification of the data at $1.1 \mathrm{~W} \mathrm{~cm}{ }^{-2}$ irradiation, while inset $\mathbf{b}$ illustrates the induced temperature increase $\triangle T$ of glass sample G5A2B as a function of laser power density.

parameters of the surrounding medium. Importantly for potential applications, within the considered range of illumination power density, a linear correlation $(R=$ 0.995) was observed between induced temperature increase and power density (inset (b) of Fig. 2).

\section{Extension of PT effect to Bi-doped silicate glasses}

Obviously, the germanate glass studied thus far is only a model material, as it does not provide the bioactivity, such as HAp precipitation, that is required for bone repair ${ }^{43}$. Next, we extended the study from germanates to the more technically relevant group of silicates. A series of Bi-doped silicate glasses were made, and the melting conditions and sample codes are compiled in Table S1. Upon laser irradiation $\left(808 \mathrm{~nm}, 1.5 \mathrm{~W} \mathrm{~cm}^{-2}\right)$, steady-state temperatures of $\sim 91{ }^{\circ} \mathrm{C}, 163^{\circ} \mathrm{C}$, and $170^{\circ} \mathrm{C}$ were reached (Fig. 3a). This indicates that the PT effect can be extended to Bi-doped silicate glasses. We also observed that in the corresponding $\mathrm{Al}_{2} \mathrm{O}_{3}$-free samples, this temperature was $\sim 20 \mathrm{~K}$ higher (Fig. 3a).

\section{Strategies to improve PT efficiency Quenching luminescence}

As noted in the previous section, the PT effect of Bidoped germanate glasses arose from the strong absorption bands in the NIR spectral region. As samples were irradiated, active species of $\mathrm{Bi}$ within them absorbed these photon energies and then enabled transition to higher excited states. Subsequent relaxation can occur through radiative and non-radiative contributions (Fig. 3b). The latter is associated with energy transfer among $\mathrm{Bi}$ ions, multiphonon relaxation, or both, which result in heat generation. Therefore, the thermal effect should be enhanced once PL is suppressed. At high concentrations of $\mathrm{Bi}_{2} \mathrm{O}_{3}$, the $\mathrm{Bi}$ centers are sufficiently close to allow inter-ionic energy transfer and, thus, luminescence quenching $^{44}$. In the $\mathrm{G} 5 \mathrm{AxB}$ system, this is clearly demonstrated by increasing the concentration of $\mathrm{Bi}_{2} \mathrm{O}_{3}$. The corresponding NIR PL emission spectra from these lower-valent $\mathrm{Bi}$ ions are shown in Fig. $4 \mathrm{a}^{42}$. As expected, the emission intensity decreases monotonically with increasing concentrations of $\mathrm{Bi}_{2} \mathrm{O}_{3}$, with a stretchedexponential correlation. This is a clear sign of quenching induced by concentration, which is further supported by the dramatic reduction of lifetime from $320.9 \mu$ s to 126.5 $\mu$ s (Fig. $4 \mathrm{a}-\mathrm{d}$ ). At the same time, emission peaks shift from $1248 \mathrm{~nm}$ to $1408 \mathrm{~nm}$, and the red-shift is possibly due to enhanced reabsorption. As Figs. 1 and 4a illustrate, the absorption spectra overlap with the emission spectra. The shift might also be influenced by the formation of different emission centers, as illustrated by the excitation spectra (Fig. 4e). Corresponding to luminescence quenching, we expect a parallel increase in the thermal contribution of the relaxation reaction. This is shown in Fig. 4f and g. The observed trends are similar to previous observations of G5A2B (see Fig. 2). For the highest doping concentration $\left(10 \mathrm{~mol} \%\right.$ of $\left.\mathrm{Bi}_{2} \mathrm{O}_{3}\right)$, the highest temperature of $\sim 102^{\circ} \mathrm{C}$ was reached (for a power density of $1 \mathrm{~W}$ $\mathrm{cm}^{-2}$ ). As observed from the thermographs, the hottest spot was typically in the center of the irradiated region. With increasing $\mathrm{Bi}_{2} \mathrm{O}_{3}$ concentrations beyond $\sim 0.5 \mathrm{~mol} \%$, the maximum temperature did not vary notably, but the heated volume increased (Fig. 4g). This is a logical result of the increasing heat generation. The concrete temperature gradient is a simple result of thermal conductivity and heat exchange at the sample surface. The change in luminescence between the samples does not provide direct information about variations in PT efficiency because the absorption coefficient at $808 \mathrm{~nm}$ also increases with $\mathrm{Bi}$ concentration. Therefore, the data were 

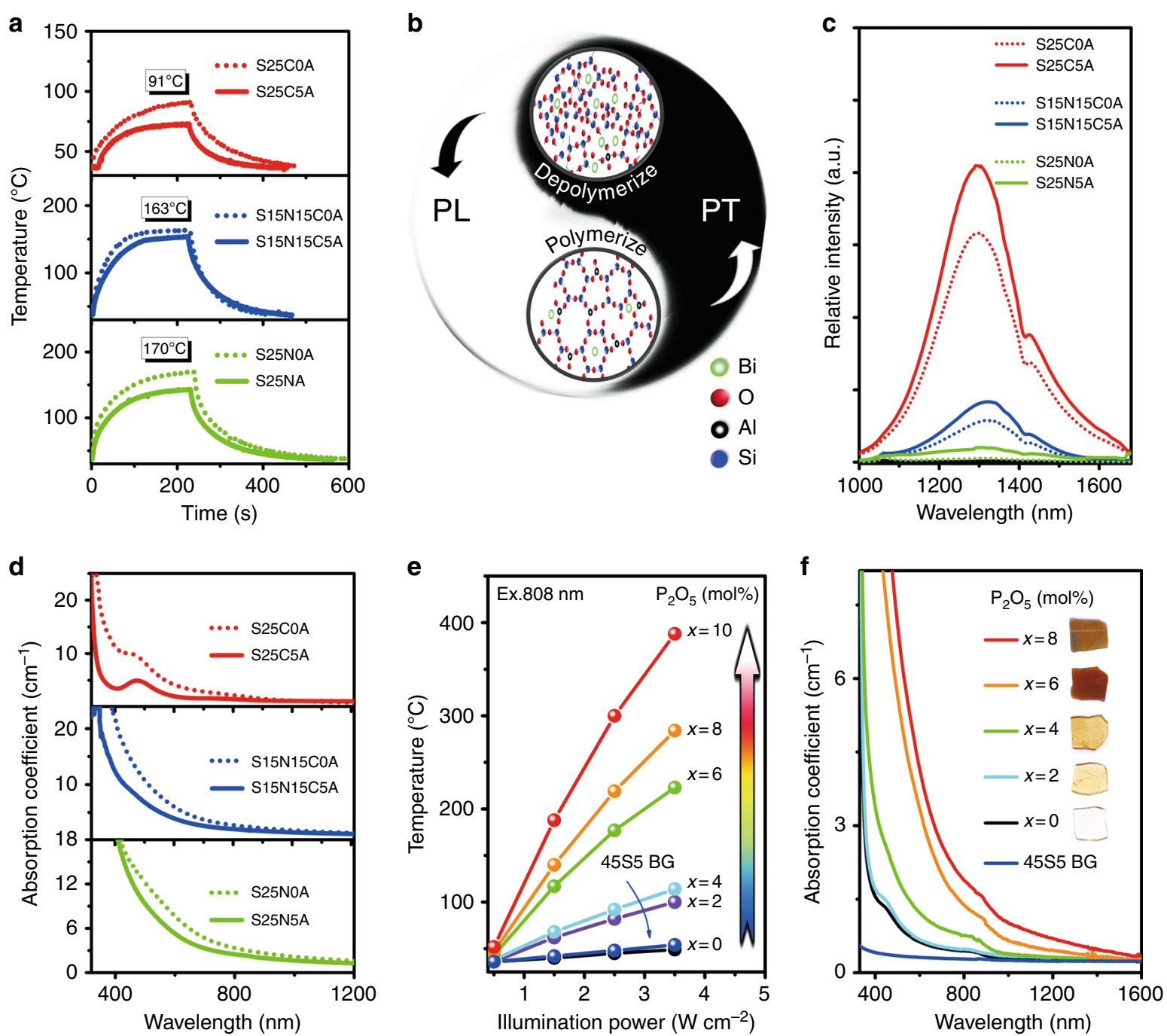

Fig. 3 Extension of PT effect to Bi-doped silicate glass, where PT effect could be enhanced by depolymerizing the glass network, and invention of Bi-doped phosphosilicate BGs with pronounced PT effect. a Temperature curves of Bi-doped silicate glass samples S25CXA, S15N15CXA, and S25NxA (for sample codes, see Table S1) under $1.5 \mathrm{~W} \mathrm{~cm}^{-2}$ irradiation of $808 \mathrm{~nm} \mathrm{LD;}$ b strategy to modulate PT efficiency; c emission spectra upon an excitation of $808 \mathrm{~nm}$; $\mathbf{d}$ absorption spectra of S25CXA, S15N15CXA, and S25NxA; e PT effect of Bi-doped phosphosilicate BG samples $\mathrm{S} \times \mathrm{P} 2 \mathrm{~B}$ as a function of illumination power and the content $\mathrm{x}$ of $\mathrm{P}_{2} \mathrm{O}_{5}$; and $\mathbf{f}$ absorption spectra of SxP2B and commercial BG 4555 .

normalized to the absorption coefficient at $808 \mathrm{~nm}$, as shown in the inset of Fig. 4b. Clearly, it deviates from a constant line, implying a nonlinear conversion from PL to thermal relaxation as the $\mathrm{Bi}$ concentration increases. Although a lower concentration of Bi can lead to a higher normalized heating efficiency, the weak absorption of samples with lower concentrations of $\mathrm{Bi}$ cannot fully absorb the incident light, preventing full utilization of the input energy. Thus, illumination only produces the lowest temperature increase for the $0.05 \mathrm{~mol} \%$ of $\mathrm{Bi}_{2} \mathrm{O}_{3}$ sample. A trade-off must be considered between heating efficiency and absorption coefficient to reach a desirable temperature increase.
Clearly, in Bi-doped germanate glasses, quenching luminescence is an effective strategy to improve the PT efficiency, and it also works well in Bi-doped silicate glasses. For instance, Bi-doped sodium calcium silicate glasses, under irradiation by $808 \mathrm{~nm} \mathrm{LD}$ with $0.9 \mathrm{~W} \mathrm{~cm}{ }^{-2}$, the sample temperature increases from $79^{\circ} \mathrm{C}$ to $162^{\circ} \mathrm{C}$ as $\mathrm{Bi}_{2} \mathrm{O}_{3}$ content increases from $1 \mathrm{~mol} \%$ to $4 \mathrm{~mol} \%$.

\section{Depolymerizing network}

According to previous studies, Bi PL strongly depends on glass network integrity, since higher polymerization can efficiently isolate $\mathrm{Bi}$ from the surroundings, leading to stronger $\mathrm{PL}^{45}$. A backbone network of silicate glass in 

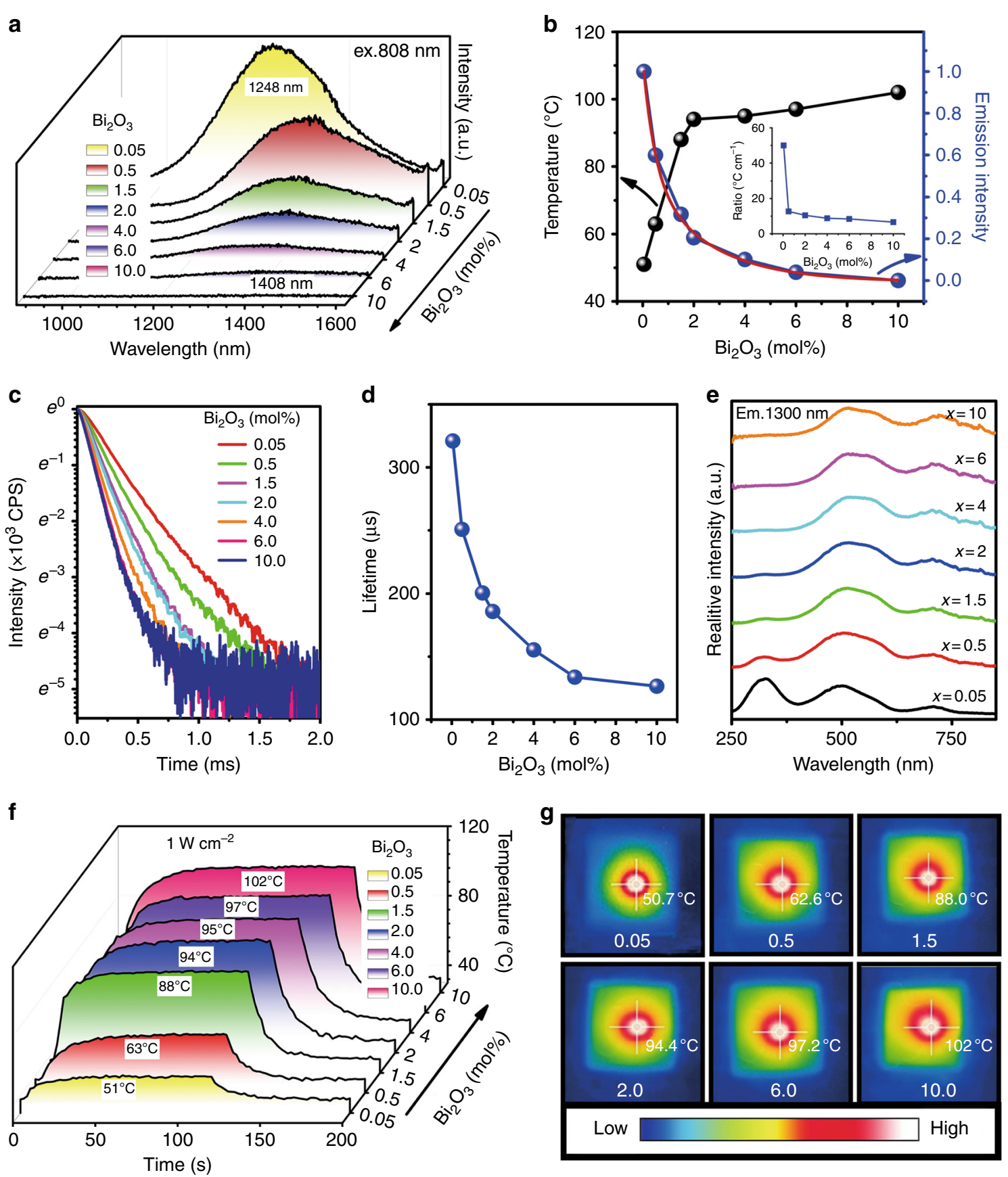

Fig. 4 Enhanced PT effect by quenching luminescence in Bi-doped germanate glass. a Emission spectra of glass samples $G 5 \mathrm{AxB}$, where the $\mathrm{Bi}_{2} \mathrm{O}_{3}$ content $x$ changes from 0.05 to 10 mol\%; $\mathbf{b}$ temperature (black line) and integrated emission intensity (blue line) of glass samples G5AxB; the red line in $\mathbf{b}$ demonstrates the double exponential fitting curve with a correlation coefficient of $99.5 \%$; the inset in $\mathbf{b}$ demonstrates the ratio of induced temperature increase $(\Delta T)$ to absorption coefficient at $808 \mathrm{~nm}$ (taken from Fig. 1) as a function of $\mathrm{Bi}_{2} \mathrm{O}_{3}$ concentration; c decay curves of $\mathrm{G} 5 \mathrm{AxB}$; $\mathbf{d}$ lifetime of G5AxB with emission at $1300 \mathrm{~nm}$ upon $808 \mathrm{~nm}$; e excitation spectra $\left(\lambda_{\mathrm{em}}=1300 \mathrm{~nm}\right.$ ) of G5AxB; $\mathbf{f}$ temperature curves of G5AxB as a function of irradiation time; power density of $808 \mathrm{~nm} \mathrm{LD}$ was attenuated to $1 \mathrm{~W} \mathrm{~cm}^{-2}$; and $\mathbf{g}$ thermal images of G5AxB, as the sample temperature became saturated.

which $\mathrm{Bi}$ resides after doping is constructed by cornersharing $\mathrm{SiO}_{4}$ tetrahedra. The polymerization can be modulated by the addition of species such as $\mathrm{Na}_{2} \mathrm{O}, \mathrm{CaO}$, or $\mathrm{Al}_{2} \mathrm{O}_{3}{ }^{46-48}$. Considering the trade-off between PL and
PT, here, we investigate the effect of these species on PT. As Fig. 3a depicts, glass samples with no aluminum, but including sodium, exhibit a higher PT effect upon the same illumination than samples with aluminum and/or 
calcium. Correspondingly, PL becomes much weaker or is quenched by, for example, sample S25N0AS. Therefore, with respect to PT efficiency, a higher content of $\mathrm{Na}_{2} \mathrm{O}$ and the absence of $\mathrm{Al}_{2} \mathrm{O}_{3}$ appear to be most beneficial. To understand this phenomenon, we measured the absorption spectra (Fig. 3d). Samples S25CxAS, which do not contain $\mathrm{Na}_{2} \mathrm{O}$, show the strongest absorption at $\sim 470 \mathrm{~nm}$, and the absorption at $700 \mathrm{~nm}$ originally observed in germanate glass emerges now in the tail of $\sim 470 \mathrm{~nm}$ absorption peak. When alumina is added, the absorption coefficients decrease, similar to a previous study on NIR $\mathrm{PL}^{49}$. This is partially due to $\mathrm{Bi}$ oxidation by an increase in melt basicity. The addition of $\mathrm{Al}_{2} \mathrm{O}_{3}$, as solid state ${ }^{27} \mathrm{Al}$ NMR spectra demonstrate, can increase network connectivity; thereby, it can topologically isolate $\mathrm{Bi}$ species from each other ${ }^{45}$. This increases the probability of radiative transitions and, in turn, suppresses PT efficiency. Thus, alumina is not suggested for the design of glass with better PT performance, and it is not considered in subsequent experiments. For glass samples free of alumina, we found that the absorption coefficient at $808 \mathrm{~nm}$ increases slightly with increasing $\mathrm{Na}_{2} \mathrm{O}$ from 2.09 to $2.43 \mathrm{~cm}^{-1}$, for example, for samples $\mathrm{S} 25 \mathrm{COA}$ and S25N0A, respectively. This is a reason that the sodium samples present a better PT property (Fig. 3a). From the microstructural point of view, introduction of sodium can efficiently depolymerize the glass network by forming non-bridged oxygen. This clearly facilitates the processes of PT and, therefore, extinguishes PL, as Fig. 3a-c illustrates.

\section{Invention of Bi-doped phosphosilicate BGs with pronounced PT effects}

As Fig. 3a demonstrates, glass sample S25N0A exhibits the best PT effect. Then, we designed a series of glass samples SxPyB (Table S1) by adding calcium and phosphorus, as these are necessary elements to regenerate bone tissues ${ }^{43}$. We sought samples with functionality in killing residual tumor cells and, consequently, promoting bone repair by remineralization of hexagonal HAp of $\mathrm{Ca}_{10}\left(\mathrm{PO}_{4}\right)_{6}(\mathrm{OH})_{2}$. During glass design, alumina was intentionally omitted due to its counteractive contribution to PT efficiency (as noted above) and potential biological toxicity, and the content of sodium was very close to S25N0A. Calcium and phosphorus were designed to replace $\mathrm{SiO}_{2}$ and therefore reduce the content of glass former ions. Therefore, the glass network can be further depolymerized, and the effect of PT can then be enhanced as Fig. $3 \mathrm{~b}$ demonstrates. The contents of phosphorus and $\mathrm{Bi}$ are modulated slightly, as Table S1 illustrates, to achieve the best compromise between PT and bioactivity.

As shown in Fig. 3e, PT heating becomes increasingly efficient with increasing $\mathrm{P}_{2} \mathrm{O}_{5}$, reaching a steady-state temperature of $\sim 188^{\circ} \mathrm{C}$ at a power density of $1.5 \mathrm{~W} \mathrm{~cm}^{-2}$ for $10 \mathrm{~mol} \%$ of $\mathrm{P}_{2} \mathrm{O}_{5}$. This effect can be readily explained by absorption spectra (Fig. 3f), where increasing $\mathrm{P}_{2} \mathrm{O}_{5}$ leads to strongly increasing absorption coefficients, with samples literally becoming black. This occurs because of increasing acidity of melts upon phosphate addition, leading to a reduction of $\mathrm{Bi}$ to the lower or even metallic state. Generally, the sample temperature increases rapidly as the power density increases (Fig. 3e). For instance, the temperature of a sample containing $10 \% \mathrm{P}_{2} \mathrm{O}_{5}$ can reach $388^{\circ} \mathrm{C}$ in air at $3.5 \mathrm{~W} \mathrm{~cm}^{-2}$. Such a significant temperature increase does not appear in commercial BG 45S5 (blue curve in Fig. 3e). The tight dependence of the PT effect on the glass compositions and incident power density reveals that it is very convenient to achieve proper temperatures by simply adjusting these parameters. This implies that less energy is needed for our glasses to reach a temperature sufficient to kill bone tumor cells due to improved PT efficiency after glass design.

\section{In vitro and in vivo biological experiments with Bi-doped BGs \\ In vitro biocompatibility of Bi-doped BGs to normal and tumor cells}

As a first step towards possible biomedical consideration, the potential toxicity of the glasses must be evaluated, especially after Bi doping. Mouse fibroblast cell line (L929), murine calvarial pre-osteoblast (MC3T3-E1) cells, rat osteosarcoma-derived (UMR106) cells, and human osteosarcoma (U2OS) cells were selected as representatives for normal and bone tumor cells for the study on biocompatibility. Generally, the cell viability (\%) was $>80$ $\%$ for the four types of cells, L929, MC3T3-E1, UMR106, and U2OS, after culturing with different concentrations of S6PyB glass for $24 \mathrm{~h}$ (Fig. 5a,b). The cell viability decreased slightly compared to that of control samples as the suspension concentration of S6PyB glass powders increased from $31.25 \mathrm{mg}^{-1} \mathrm{~L}^{-1}$ to $1000 \mathrm{mg}^{-1}$. For glass sample $\mathrm{S} 6 \mathrm{P} 6 \mathrm{~B}$, where the $\mathrm{Bi}_{2} \mathrm{O}_{3}$ concentration was $6 \mathrm{~mol}$ $\%$, the cell viability was found to decrease from $81 \%$ to 71 $\%$ as the power suspension concentration increased to

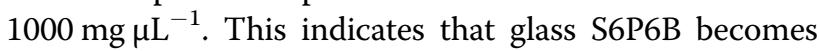
toxic for L929 cells. Therefore, in the following, the sample with $y=6 \mathrm{~mol} \%$ was not further considered.

\section{In vitro mineralization of Bi-doped BG samples S6PXB in SBF solution}

To test the bioactivity of the present material, we first examined HAp mineralization during exposure to SBF. Figure 5d depicts field emission scanning electron microscopy (SEM) micrographs of the mineral deposit, which was found on the material surface after immersion in SBF. A rod-like morphology (with a rod diameter of $\sim 50 \mathrm{~nm}$ ) was observed on the surface of the S6PyB samples $(y=0,1 \mathrm{~mol} \%)$ after 3 days in SBF. There was no 

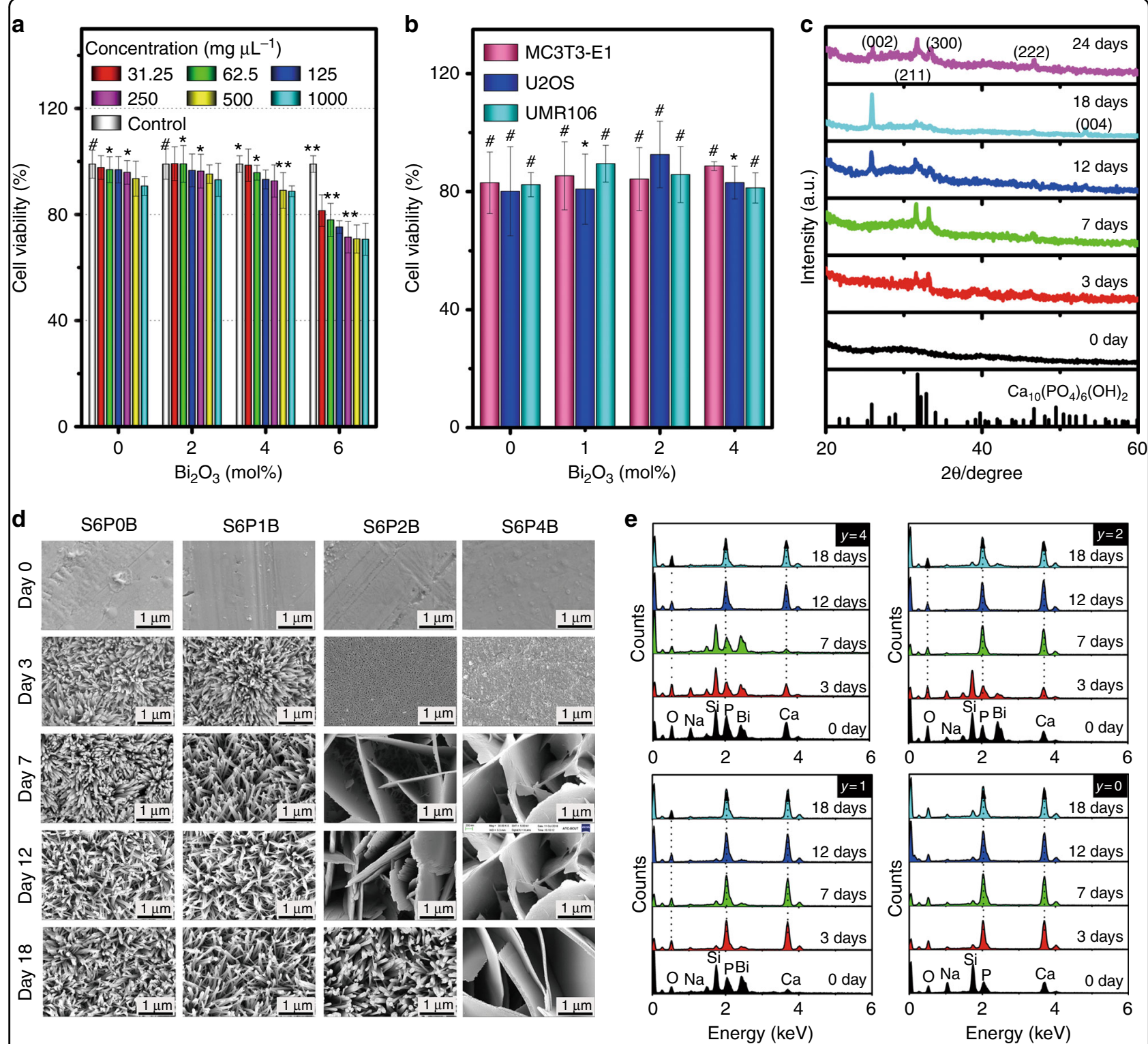

Fig. 5 In vitro biocompatibility and mineralization of Bi-doped BG samples S6PyB in SBF solution. a Viability of mouse fibroblast cell line L929

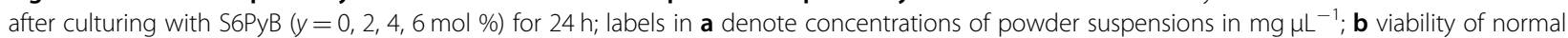
cell MC3T3-E1, and tumor cells of human osteosarcoma line U2OS and rat osteosarcoma cell lines UMR106 after co-culturing with S6PyB; data points represent the mean values and error bars according to three independent experiments; c XRD patterns of sample S6P2B after immersion in SBF for different times as indicated, standard data of JCPDS Card No. 03-0747 of $\mathrm{Ca}_{10}\left(\mathrm{PO}_{4}\right)_{6}(\mathrm{OH})_{2}(\mathrm{HAp})$ is listed as reference at the bottom; d morphological evolution of glass samples S6PyB as incubated in SBF for different days as indicated; e evolution of element redistribution on sample surfaces of S6PyB as incubated in SBF for different days.

visible difference between these two samples for all exposure times, that is, in the absence of $\mathrm{Bi}_{2} \mathrm{O}_{3}$ or for a $\mathrm{Bi}_{2} \mathrm{O}_{3}$ content of $1 \mathrm{~mol} \%$. At higher Bi content, significant differences were observed between the samples. That is, for $y=2-4 \mathrm{~mol} \%$, the initial porous microstructure (3 days immersion) transforms into an assembly of platelets after 7 days of exposure. The rod-shaped morphology was observed only after 12 days or never for $y=$ $4 \mathrm{~mol} \%$. Clearly, the larger surface area of the rod-shaped clusters accelerates the corrosion reaction relative to the platelet morphology.

The corrosion reaction occurs for Bi-doped BGs, as such glasses are soaked in SBF solution. Once corrosion starts, the glass surface turns from smooth to porous or rough, which is followed by precipitation and growth of a new phase, that is, $\mathrm{HAp} \mathrm{Ca}_{10}\left(\mathrm{PO}_{4}\right)_{6}(\mathrm{OH})_{2}$ (Fig. 5c, d). As soon as the phase of HAp appears and anchors on the sample surface, elements such as $\mathrm{P}, \mathrm{Ca}$, and $\mathrm{O}$ will diffuse 


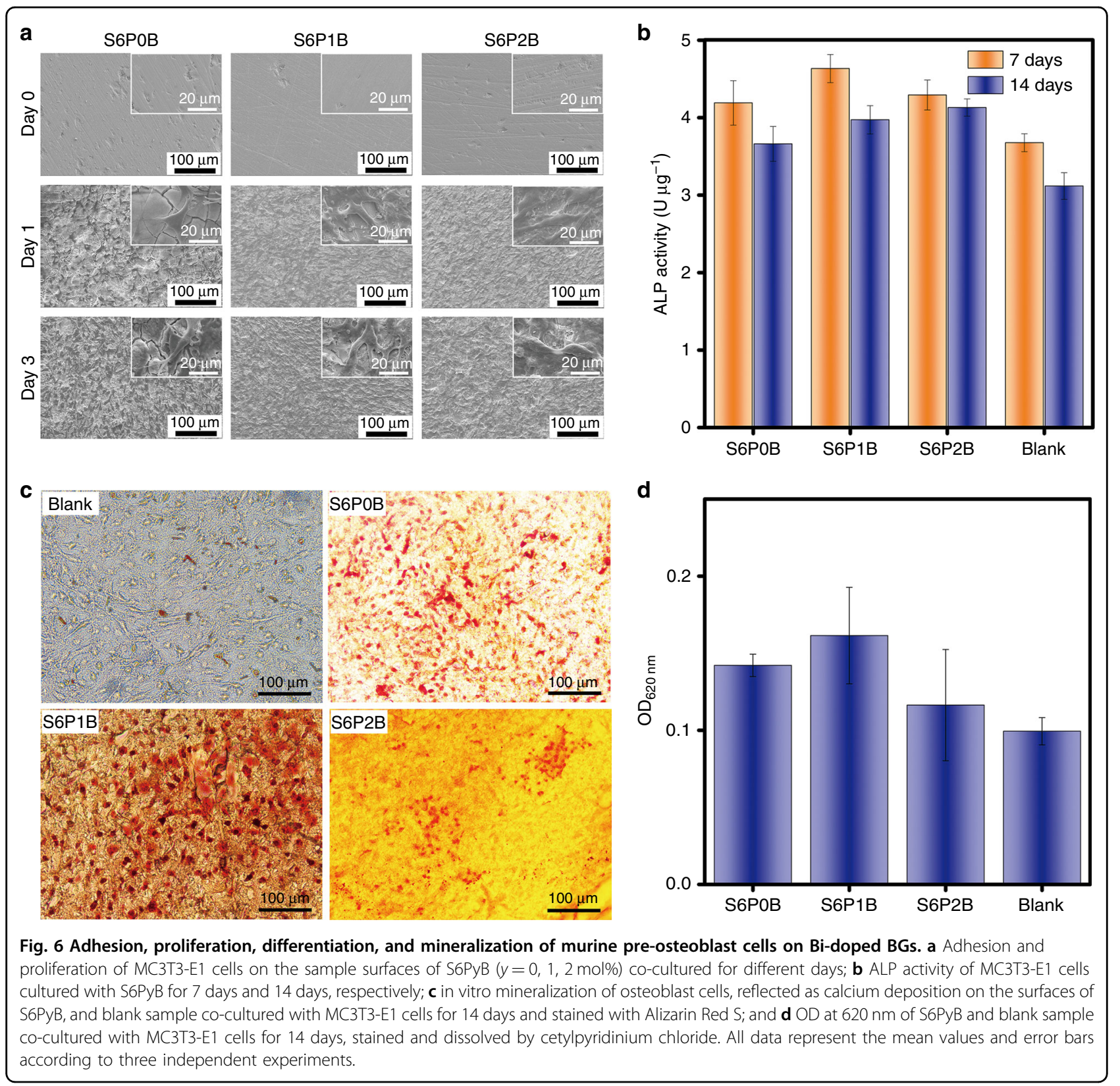

in the vicinity from either SBF solution or glass, promoting further growth of HAp specifically and the mineralization processes generally (Fig. 5c, e). The corrosion reaction, which usually destroys the glass surface and leads to the loss of elements, threatens longevity of the BGs, but it induces bone regeneration and repair.

The results of energy-dispersive X-ray spectroscopy (EDS) analyses are shown in Fig. 5e. After 3 days of immersion, $\mathrm{P}$ and $\mathrm{Ca}$ are the primary cation species found in the surface deposit, with significant traces of the other glass components still present, especially for the higher content, $y$, of Bi. After 7 days of incubation, the traces of residual glass practically disappear, which indicates the formation of HAp. The addition of $\mathrm{Bi}_{2} \mathrm{O}_{3}$ appears to delay this corrosion and remineralization reaction to a certain extent. The delay enables the precipitation of larger platelets, thereby promoting the incidence of light onto the sample surface through the spaces between these platelets (Fig. 5d). This eventually leads to an improved PT effect compared to the blank sample, where light propagation is blocked by the dense rod assembly covered on the surface (Fig. 5d). Such PT effects can persist even as the samples have been soaked in SBF for 30 days, although it is slightly weakened as the soaking time is prolonged (Figure S2). This should be due to the formation of a HAp layer coated 
on the sample surface, which may attenuate incident light. The enhanced and persistent PT effect will efficiently and completely extinguish residual bone tumors in the initial state of mineralization. At a later state, platelets of HAp evolve into densely assembled rods on the sample surface, and this strengthens the regenerated bone tissue (see Fig. $5 \mathrm{~d}$ for the SEM image for $2 \mathrm{~mol} \% \mathrm{Bi}_{2} \mathrm{O}_{3}$ sample soaked in SBF for 18 days).

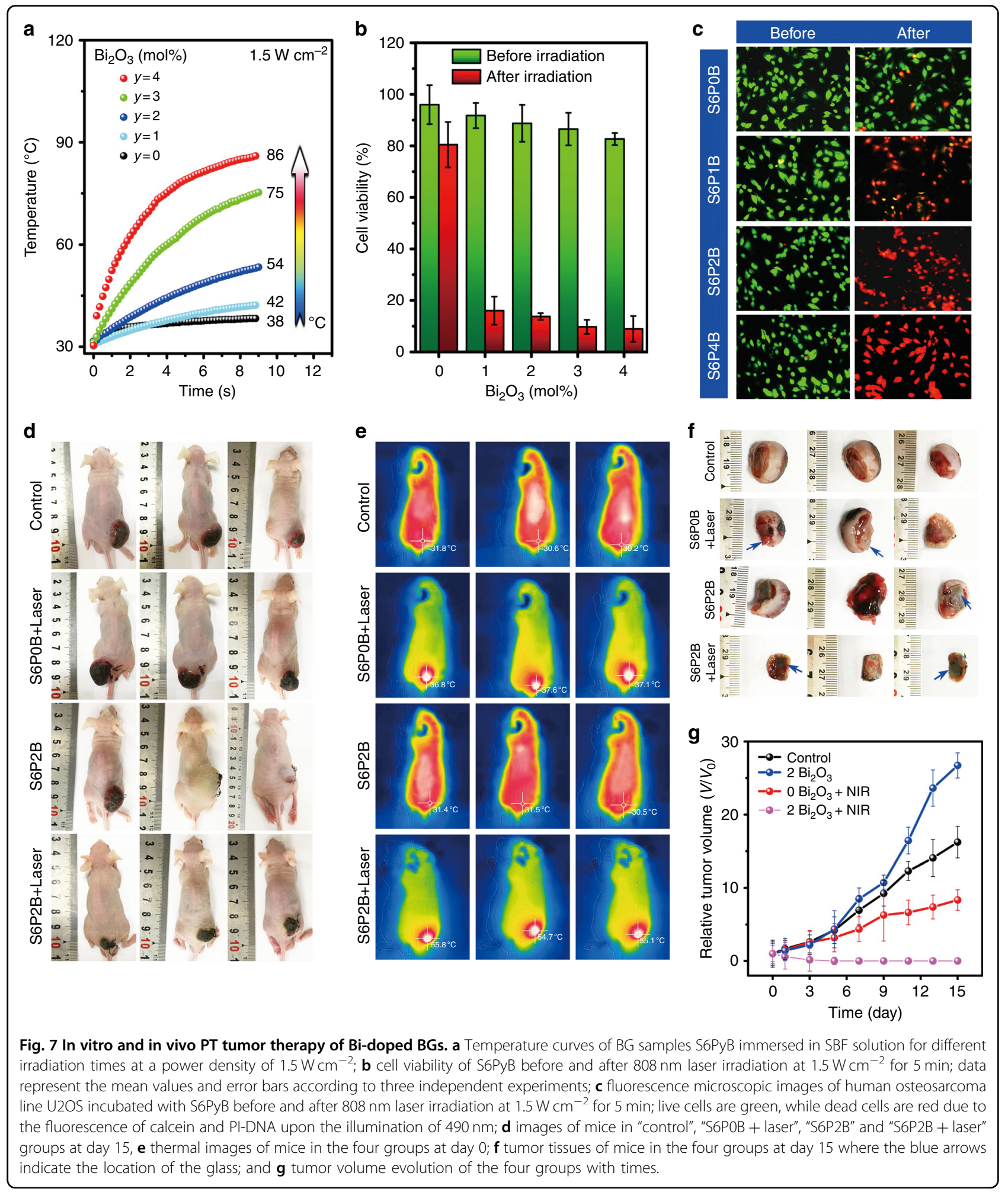




\section{Adhesion, proliferation, differentiation, and mineralization of murine pre-osteoblast cells on Bi-doped BGs}

In addition to biocompatibility, Bi-doped BGs should enable pre-osteoblast cells to land, populate and mineralize into bone cells, which will eventually regenerate and repair the bone tissues ${ }^{10}$. Therefore, we chose MC3T3-E1 as representative pre-osteoblast cells for this study. After the cells were co-cultured with these glasses in Dulbecco's minimum essential medium (DMEM) for 1 day, more cracks appeared on the surface of sample S6P0B than on S6P1B or S6P2B. At the same time, the osteoplastic cells started to populate throughout the originally smooth surfaces of these glasses and stick tightly to them (Fig. 6a). At day 3 of co-culture, these cells proliferated quickly and the cell density increased. The surface of S6P0B also became rougher, and gaps between cracks were larger. Cracks started appearing on S6P2B, which is shown in Fig. 5d.

At the early stage of osteogenesis, alkaline phosphatase (ALP) appears in these osteoplastic cells and catalyzes bone cell differentiation ${ }^{50}$. The value of ALP can be observed in the bone cells MC3T3-E1. As Fig. 6b demonstrated, the bone cells exhibited higher ALP values on Bi-doped BGs than the blank or sample S6P0B without Bi on day 7 and day 14. For all samples, the value of ALP on day 14 was lower than that on day 7 , perhaps because the cells entered the phases of extracellular matrix maturation or mineralization for bone formation.

In vitro mineralization of osteoblast cells was indicated by calcium deposition (or nodules formed by osteogenic cells) on the surfaces of S6PyB and blank samples cocultured with MC3T3-E1 cells for 14 days and stained with Alizarin Red S (Fig. 6c). Bi-doped BG, especially $\mathrm{S} 6 \mathrm{P} 1 \mathrm{~B}$, exhibited the highest calcium deposition or the most nodules on the surface of all samples (Fig. 6c, d). As Fig. $6 \mathrm{~d}$ demonstrated, it was 1.62 times higher than the blank control, where no BG was applied.

These in vitro experiments demonstrate that the Bi-doped BGs exhibit excellent biocompatibility with pre-osteoblast cells, and they can facilitate cell proliferation and osteogenic differentiation and mineralization.

\section{In vitro PT effect of Bi-doped BGs}

We tested hyperthermia in vitro, using S6PyB $(y=0,1$, $2,4 \mathrm{~mol} \%$ ) as the PT material, in turn, with SBF, methylthiazolyldiphenyl tetrazolium bromide (MTT) assay, and calcein-AM/PI staining for live/dead staining. As Fig. 7a demonstrated, the temperature of $\mathrm{S} 6 \mathrm{PyB}$ increased from $42{ }^{\circ} \mathrm{C}$ to $86^{\circ} \mathrm{C}$ when the concentration of $\mathrm{Bi}_{2} \mathrm{O}_{3}$ in the glass increased from 1 to $4 \mathrm{~mol} \%$. In the undoped control, there is practically no temperature effect. The decrease in heating kinetics is caused by the improved heat transfer at the sample surface because of
SBF immersion. At this point, it seems very feasible to generate the desired heating effect necessary for hyperthermia applications using the present BG. Human osteosarcoma line U2OS cells were selected as the most typical bone tumor cells for PT therapy. Standard MTT assays were carried out to determine the relative viabilities after $24 \mathrm{~h}$ of incubation (Fig. $7 \mathrm{~b}$ and Figure S3). All samples exhibited non-cytotoxicity without laser irradiation. After irradiation with an $808 \mathrm{~nm}$ laser, more than 80 $\%$ of the human osteosarcoma line U2OS cells were killed by $\operatorname{S6PyB}(y=1,2,3,4 \mathrm{~mol} \%)$ samples, whereas the cell viability in the undoped control was only decreased by 15 $\%$. This effect was further investigated and confirmed by fluorescence microscopy. Clearly, cell mortality gradually increased with the concentration of $\mathrm{Bi}_{2} \mathrm{O}_{3}$. The tumor cells incubated with the $\mathrm{S} 6 \mathrm{P} 1 \mathrm{~B}$ glass sample could reactivate, but $2-4 \mathrm{~mol} \%$ of $\mathrm{Bi}_{2} \mathrm{O}_{3}$ led to destructive death (Fig. 7c). This is clear evidence for the potential of hyperthermia combined with a BG material.

\section{In vivo PT therapy experiments on nude mice}

To further demonstrate possible in vivo applications, four parallel in vivo experiments were performed with nude mice, including "control", "S6P0B + laser", "S6P2B", and "S6P2B + laser" groups. For the "control" group, no glass or laser irradiation was applied. As the rat osteosarcoma derived from UMR106 cells grew to $\sim 10 \mathrm{~mm}$, glass samples were implanted into the lower tumors of mice (Fig. 7d). Hematoxylin and eosin (H\&E) stain analyses demonstrated that implanting Bi-doped or undoped glass samples for 15 days did not lead to damage or inflammation of mice organs, such as heart, liver, spleen, lung, or kidney (Figure S4). The temperature of the tumor site lies between 30 and $32^{\circ} \mathrm{C}$, and the implanted BG did not produce a noticeable difference between groups of "control" and "S6P2B," if individual differences between mice were neglected (Fig. 7e). Regarding laser illumination of the tumors, the temperature of the "S6P0B + laser" group increased by $6-7^{\circ} \mathrm{C}$ while "S6P2B + laser" group soon reached $\sim 55^{\circ} \mathrm{C}$ (Fig. 7e). The laser illumination persisted for only $10 \mathrm{~min}$ at a power density of $1.5 \mathrm{~W}$ $\mathrm{cm}^{-2}$ before it was switched off. There was an obvious difference between these groups. Day 15 tumors in the "control", "S6P0B + laser", and "S6P2B" groups became larger (Fig. 7d, f, g), and tumor cells were alive based on the H\&E stain analyses (Figure S4). However, tumors in the "S6P2B + laser" group started disappearing on day 1 , and they vanished at day 3 . At day 15 , all tumors were destroyed (Figure S4), and the only BGs observed at original tumor sites and normal muscular tissues had already started growing on the surfaces, wrapping around them. This demonstrates in vivo biocompatibility and tumor therapy potential for such glasses. 


\section{Conclusions}

In summary, after the discovery of the PT effect in Bidoped glasses, we determined that such an effect can be effectively controlled by quenching luminescence using the active Bi species or depolymerizing the glass network. Consequently, we have developed a new type of Bi-doped bioactive phosphosilicate glass, which, as in vitro experiments demonstrate, combines high PT conversion efficiency, biocompatibility, and HAp remineralization to reduce the number of treatment cycles required to treat large bone defects. The glasses also allow the population, differentiation and mineralization of osteogenic cells on their surfaces, demonstrating proof of concept to repair bone tissues. Both in vitro and in vivo experiments indicate that these glasses can efficiently destroy bone tumor cells upon illumination with NIR light and also promote regrowth of the muscle tissue around them. H\&E stain analysis demonstrate that implanting these glasses did not permanently damage mice organs. Thus, Bi-doped BGs assist with toxic chemo-therapies and radiation-therapies, inducing the destruction of residual cancer cells, and they also enable the application of emerging treatment techniques, such as interventional treatment. This indicates an excellent potential for the development and application of multi-functional biomaterials.

\section{Acknowledgements \\ We thank Prof. Dr. Lothar Wondraczek from the University of Jena for inspiring discussions during the preparation of this manuscript. We gratefully acknowledge financial support from the Program for Innovative Research Team in University of Ministry of Education of China (Grant No. IRT_17R38), the National Natural Science Foundation of China (Grant No. 51672085), the Key Program of Guangzhou Scientific Research Special Projects (Grant No. 201607020009), the Joint Fund of Ministry of Education of China, and the Fundamental Research Funds for the Central Universities.}

\section{Author details \\ 'The State Key Laboratory of Luminescent Materials and Devices, Guangdong Engineering Technology Research and Development Center of Special Optical Fiber Materials and Devices, Guangdong Provincial Key Laboratory of Fiber Laser Materials and Applied Techniques, School of Materials Science and Engineering, South China University of Technology, 510641 Guangzhou, China. 2Department of Chemistry, Imperial College London, South Kensington, London SW7 2AZ, UK. ${ }^{3}$ Guangdong Key Lab of Orthopedic Technology and Implant Materials, Department of Orthopedics, Guangzhou General Hospital of Guangzhou Military Command, 111 Liuhua Road, 510010 Guangzhou, China. ${ }^{4}$ Department of Chemistry and Biochemistry Stephenson Life Sciences Research Center, University of Oklahoma, Norman, OK 73072, USA. ${ }^{5}$ School of Materials Science and Engineering, South China University of Technology, Guangzhou 510641, China}

\section{Conflict of interest}

The authors declare that they have no conflict of interest.

Supplementary information accompanies for this paper at https://doi.org/ 10.1038/s41377-018-0007-z.

Received: 5 December 2017 Revised: 31 January 2018 Accepted: 1 February 2018 Accepted article preview online: 11 February 2018

Published online: 18 May 2018

\section{References}

1. Currey, J. D. Bones: Structure and Mechanics (Princeton University Press, Princeton, Oxford, 2002).

2. D'Oronzo, S., Stucci, S., Tucci, M. \& Silvestris, F. Cancer treatment-induced bone loss (CTIBL): pathogenesis and clinical implications. Cancer Treat. Rev. 41, 798-808 (2015).

3. Wang, J. L. et al. Phage nanofibers induce vascularized osteogenesis in 3D printed bone scaffolds. Adv. Mater. 26, 4961-4966 (2014).

4. Chu, K. F. \& Dupuy, D. E. Thermal ablation of tumours: biological mechanisms and advances in therapy. Nat. Rev. Cancer 14, 199-208 (2014).

5. Shen, J., Zhao, L. \& Han, G. Lanthanide-doped upconverting luminescent nanoparticle platforms for optical imaging-guided drug delivery and therapy. Adv. Drug Deliv. Rev. 65, 744-755 (2013).

6. Mirza, A. N. et al. Radiofrequency ablation of solid tumors. Cancer J. 7, 95-102 (2001).

7. Kennedy, J. E. High-intensity focused ultrasound in the treatment of solid tumours. Nat. Rev. Cancer 5, 321-327 (2005).

8. Yan, J. H., Ma, C. R., Liu, Pu, Wang, C. X. \& Yang, G. W. Generating scattering dark states through the Fano interference between excitons and an individual silicon nanogroove. Light Sci. Appl. 6, e16197 (2017).

9. Ikenaga, M. et al. Localized hyperthermic treatment of experimental bone tumors with ferromagnetic ceramics. J. Orthop. Res 11, 849-855 (1993).

10. Hench, L. L. \& Kokubo, T. in Handbook of Biomaterial Properties (eds Black, J. \& Hastings, G.) (Springer, Bostan, MA, 1998).

11. Jiang, Y. M., Ou, J., Zhang, Z. H. \& Qin, Q. H. Preparation of magnetic and bioactive calcium zinc iron silicon oxide composite for hyperthermia treatment of bone cancer and repair of bone defects. J. Mater. Sci. Mater. Med. 22, 721-729 (2011).

12. Bretcanu, $\mathrm{O}$. et al. The influence of crystallised $\mathrm{Fe}_{3} \mathrm{O}_{4}$ on the magnetic properties of coprecipitation-derived ferrimagnetic glass-ceramics. Acta Biomater. 1, 421-429 (2005).

13. Xiao, J. W. et al. Porous Pd nanoparticles with high photothermal conversion efficiency for efficient ablation of cancer cells. Nanoscale 6, 4345-4351 (2014).

14. Liu, B. et al. Magnetically targeted delivery of DOX loaded $\mathrm{Cu}_{9} \mathrm{~S}_{5} @ \mathrm{mSiO}_{2} @-$ $\mathrm{Fe}_{3} \mathrm{O}_{4}$-PEG nanocomposites for combined MR imaging and chemo/photothermal synergistic therapy. Nanoscale 8, 12560-12569 (2016).

15. Cheng, L., Wang, C., Feng, L. Z., Yang, K. \& Liu, Z. Functional nanomaterials for phototherapies of cancer. Chem. Rev. 114, 10869-10939 (2014).

16. Son, J. et al. Ultrafast photonic PCR. Light Sci. Appl. 4, e280 (2015).

17. Ming, T. et al. Strong polarization dependence of plasmon-enhanced fluorescence on single gold nanorods. NanoLetters 9, 3896-3903 (2009).

18. Blum, O. \& Shaked, N. T. Prediction of photothermal phase signatures from arbitrary plasmonic nanoparticles and experimental verification. Light Sci. Appl. 4, e322 (2015).

19. Bakewell, C., White, A. J. P., Long, N. J. \& Williams, C. K. Metal-size influence in iso-selective lactide polymerization. Angew Chem. Int Ed. Engl. 53, 9226-9230 (2014).

20. Yang, D. et al. Assembly of Au plasmonic photothermal agent and iron oxide nanoparticles on ultrathin black phosphorus for targeted photothermal and photodynamic cancer therapy. Adv. Funct. Mater. 27, 1700371 (2017).

21. Qiu, P. H. et al. Tuning photothermal properties of gold nanodendrites for in vivo cancer therapy within a wide near infrared range by simply controlling their degree of branching. Biomaterials 104, 138-144 (2016).

22. Yang, K. et al. Graphene in mice: ultrahigh in vivo tumor uptake and efficient photothermal therapy. NanoLetters 10, 3318-3323 (2010).

23. Li, D. et al. Supra-(carbon nanodots) with a strong visible to near-infrared absorption band and efficient photothermal conversion. Light Sci. Appl. 5, e16120 (2016).

24. Qu, D. et al. Preparation of graphene nanosheets/copper composite by spark plasma sintering. Adv. Mater. Res. 833, 276-279 (2013).

25. Robinson, J. T. et al. Ultrasmall reduced graphene oxide with high nearinfrared absorbance for photothermal therapy. J. Am. Chem. Soc. 133, 6825-6831 (2011).

26. Chan, C. F. et al. pH-dependent cancer-directed photodynamic therapy by a water-soluble graphitic-phase carbon nitride-porphyrin nanoprobe. ChemPlusChem 81, 535-540 (2016).

27. Hessel, C. M. et al. Copper selenide nanocrystals for photothermal therapy. NanoLetters 11, 2560-2566 (2011).

28. Tian, Q. W. et al. Hydrophilic $\mathrm{Cu}_{9} \mathrm{~S}_{5}$ nanocrystals: a photothermal agent with a $25.7 \%$ heat conversion efficiency for photothermal ablation of cancer cells in vivo. ACS Nano 5, 9761-9771 (2011). 
29. Feng, W. et al. In vitro and in vivo toxicity studies of copper sulfide nanoplates for potential photothermal applications. Nanomed. Nanotechnol. Biol. Med. 11, 901-912 (2015).

30. Xie, R. J. \& Hintzen, H. T. Optical properties of (Oxy) nitride materials: a review. J. Am. Ceram. Soc. 96, 665-687 (2013).

31. Deng, X. R. et al. Rational design of a comprehensive cancer therapy platform using temperature-sensitive polymer grafted hollow gold nanospheres: simultaneous chemo/photothermal/photodynamic therapy triggered by a $650 \mathrm{~nm}$ laser with enhanced anti-tumor efficacy. Nanoscale 8, 6837-6850 (2016).

32. Hong, R. et al. Glutathione-mediated delivery and release using monolayer protected nanoparticle carriers. J. Am. Chem. Soc. 128, 1078-1079 (2006).

33. Shen, J. et al. Engineering the upconversion nanoparticle excitation wavelength: cascade sensitization of tri-doped upconversion colloidal nanoparticles at $800 \mathrm{~nm}$. Adv. Opt. Mater. 1, 644-650 (2013).

34. Deng, K. R. et al. $808 \mathrm{~nm}$ light responsive nanotheranostic agents based on near-infrared dye functionalized manganese ferrite for magnetic-targeted and imaging-guided photodynamic/photothermal therapy. J. Mater. Chem. B 5, 1803-1814 (2017)

35. Wu, C. T., Chang, J., Wang, J. Y., Ni, S. Y. \& Zhai, W. Y. Preparation and characteristics of a calcium magnesium silicate (bredigite) bioactive ceramic. Biomaterials 26, 2925-2931 (2005).

36. Lee, J. W., Yamaguchi, A. \& limura, T. Functional heterogeneity of osteocytes in FGF23 production: the possible involvement of DMP1 as a direct negative regulator. Bone Rep. 3, 543 (2014).

37. Qin, G. et al. Melittin inhibits tumor angiogenesis modulated by endothelial progenitor cells associated with the SDF-1a/CXCR4 signaling pathway in a UMR-106 osteosarcoma xenograft mouse model. Mol. Med Rep. 14, 57-68 (2016).

38. Bartkova, J. et al. Oncogene-induced senescence is part of the tumorigenesis barrier imposed by DNA damage checkpoints. Nature $\mathbf{4 4 4}$ 633-637 (2006).
39. Zhao, Z. X. et al. Highly selective mitochondria-targeting amphiphilic silicon(IV) phthalocyanines with axially ligated rhodamine B for photodynamic therapy. Inorg. Chem. 51, 812-821 (2011).

40. Jin, M. Z., Lu, F. \& Belkin, M. A. High-sensitivity infrared vibrational nanospectroscopy in water. Light Sci. Appl. 6, e17096 (2017).

41. Zheng, J. Y. et al. Broadband NIR luminescence from a new bismuth doped $\mathrm{Ba}_{2} \mathrm{~B}_{5} \mathrm{O}_{9} \mathrm{Cl}$ crystal: evidence for the $\mathrm{Bi}^{0}$ model. Opt. Express 20, 22569-22578 (2012).

42. Peng, M. Y., Zollfrank, C. \& Wondraczek, L. Origin of broad NIR photoluminescence in bismuthate glass and Bi-doped glasses at room temperature. J. Phys. Condens Matter 21, 285106 (2009).

43. Hench, L. L. in Handbook of Bioceramics and Biocomposites (ed I. Antoniac, I.) 23-33 (Springer, Cham, 2016).

44. Zhao, Y. Q., Peng, M. Y., Mermet, A., Zheng, J. Y. \& Qiu, J. R. Precise frequency shift of NIR luminescence from bismuth-doped $\mathrm{Ta}_{2} \mathrm{O}_{5}-\mathrm{GeO}_{2}$ glass via composition modulation. J. Mater. Chem. C. 2, 7830-7835 (2014).

45. Wang, L. P., Tan, L. L., Yue, Y. Z., Peng, M. Y. \& Qiu, J. R. Efficient enhancement of bismuth NIR luminescence by aluminum and its mechanism in bismuthdoped germanate laser glass. J. Am. Ceram. Soc. 99, 2071-2076 (2016).

46. Stebbins, J. F. \& Xu, Z. NMR evidence for excess non-bridging oxygen in an aluminosilicate glass. Nature 390, 60-62 (1997).

47. Neuville, D. R., Cormier, L., Montouillout, V. \& Massiot, D. Local Al site distribution in aluminosilicate glasses by ${ }^{27}$ Al MQMAS NMR. J. Non-Cryst. Solids 353, 180-184 (2007).

48. Kjeldsen, J. et al. Mixed alkaline earth effect in sodium aluminosilicate glasses. J. Non-Cryst. Solids 369, 61-68 (2013).

49. Nielsen, K. H., Smedskjaer, M. M., Peng, M. Y., Yue, Y. Z. \& Wondraczek, L. Surface-luminescence from thermally reduced bismuth-doped sodium aluminosilicate glasses. J. Non-Cryst. Solids 358, 3193-3199 (2012).

50. Li, M. et al. Stimulatory effects of the degradation products from Mg-Ca-Sr alloy on the osteogenesis through regulating ERK signaling pathway. Sci. Rep. 6, 32323 (2016) 